\title{
32. A HIGH-RESOLUTION STUDY OF THE OUTER PARTS OF THE GALAXY
}

\author{
B. F. Burke, K. C. Turner, and M. A. Tuve
}

Carnegie Institution of Washington, Department of Terrestrial Magnetism

\section{Introduction}

The 300-foot transit telescope at the N.R.A.O. at Green Bank, West Virginia, was used in conjunction with the Carnegie 90-channel $\mathrm{H}$-line spectrograph to study the far parts of the Galaxy between $l=11^{\circ}$ and $l=50^{\circ}\left(l^{\mathrm{II}}\right.$ and $b^{\mathrm{II}}$ are to be understood throughout). A travelling feed was constructed at the Carnegie Institution which enabled us to track one point in the sky for approximately $5^{\mathrm{m}} \mathbf{4 0}^{\mathrm{s}}$. This made it possible to make two completely independent frequency scans of a given point each day. On successive days, duplicate curves were taken with the frequency scale shifted, in order to guard against single channel anomalies and pointing error. Line profiles were taken at 160 points in the region under study, in meridional sections every $5^{\circ}$ of $l$, from $b=-0.8$ to $b=+2.0$ in steps of 0.2 . In addition, a single longitudinal section was taken at $b=+0.4$ in steps of $1^{\circ}$.

\section{The Instrument}

At $21 \mathrm{~cm}$ the beam of the 300 -foot telescope has a width between the half-power points of $10 \mathrm{~min}$ of arc. At declination $0^{\circ}$, a point in the sky travels this distance in $40 \mathrm{sec}$. The noise figure of the 90 -channel spectrograph (about $500^{\circ} \mathrm{K}$ double sideband) is such that a $2 \frac{1}{2}$ min integration time is most useful. In order to resolve this conflict, a movable feed was constructed at the Department of Terrestrial Magnetism, designed by Mr. E. T. Ecklund. This moved a dipole feed across the focal plane in such a way as to track the image of the object of interest. Aberrations limited the distance of travel to about four beamwidths on either side of the axis. This made available an integration time of $5 \mathrm{~min}$ and $40 \mathrm{sec}$, just $1 \mathrm{~min}$ less than the time required to run through the 90 channels two times. This 1 min difference, together with a $15 \%$ error in track rate discovered at the end of the observing period (because simple angle equivalents are not preserved for a dipole off axis in a parabola), broadened the beam in right ascension by $\mathbf{3}^{\prime} .6 \cos \delta$.

The data to be discussed in this paper come from the 53 narrow-band channels ( $10 \mathrm{kc} / \mathrm{s}$ wide) of the Carnegie $\mathrm{H}$-line spectrometer. (The $50 \mathrm{kc} / \mathrm{s}$ bandwidth of the other channels made them useful primarily as a control.) The gains of the individual channels were checked at least once a day for uniformity with a neon bulb noise source located at the feed. As a rule the gains remained the same to within about $2 \%$, although an occasional adjustment of 5-10\% would be required for one channel or another. The overall sensitivity of the instrument was checked each day by taking a profile at a standard point in the sky: $l^{\mathrm{I}}=50^{\circ}, b^{\mathrm{I}}=0^{\circ}$. The height of the main peak (defined as 100 intensity units) was constant within about $5 \%$ over the observing period of a month. 


\section{The Observations}

From the line profiles produced by the spectrograph, contour maps were drawn of nine meridional sections at $l=15^{\circ}$ to $l=50^{\circ}$ at $5^{\circ}$ intervals. The individual

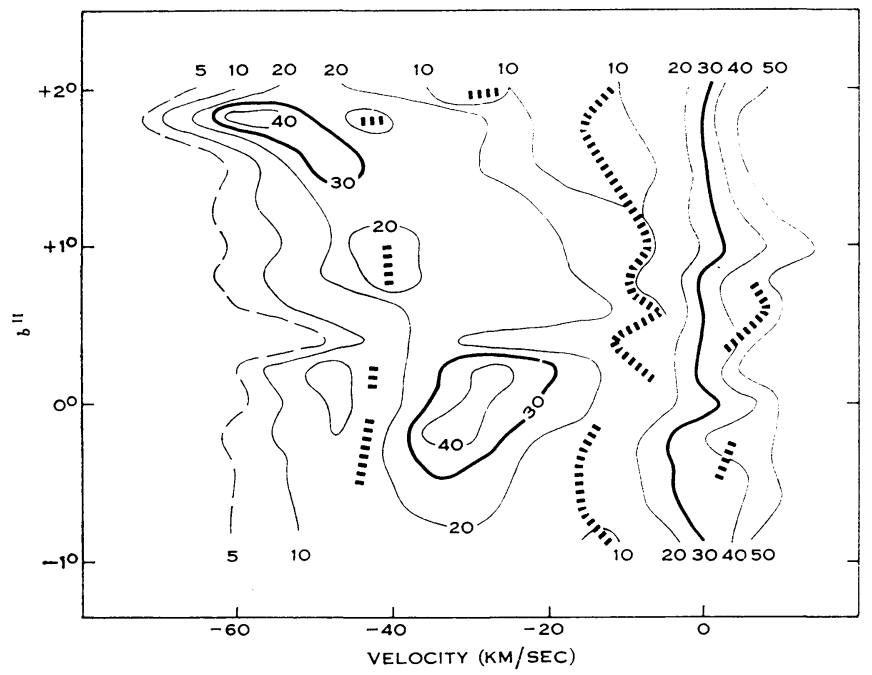

Fig. 1.-Observed antenna temperature along $l=35^{\circ}$. Velocity is with respect to the local standard of rest.

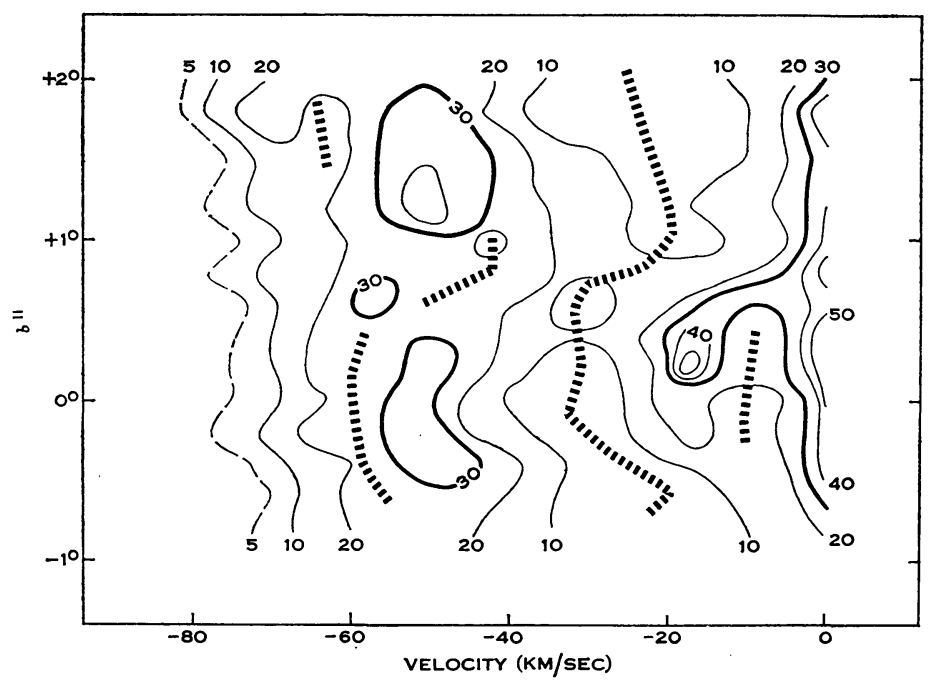

Fig. 2.- Observed antenna temperature along $l=50^{\circ}$. Velocity is with respect to the local standard of rest.

profiles in each section were taken every $12^{\prime}$ in $b$, except for the section at $l=11^{\circ}$, where some profiles were taken every $6^{\prime}$. Sample maps are shown in Figures 1 and 2. In addition, a longitudinal section between $l=11^{\circ}$ and $l=54^{\circ}$ was taken at $b=+0.4$, with individual profiles separated by $1^{\circ}$ in $l$. This is shown in Figure 3, with the 
"valleys" (minimum hydrogen) marked by the dashed lines. On examination of these maps certain principal features may be noted:

(a) The hydrogen distribution is very complex. The size of the structures observed runs from $2^{\circ}$ to 0.2 . Figure 1 shows several such structures. The concentration centred at $v_{r}=-30 \mathrm{~km} / \mathrm{sec}, b=0.0$, has a half-brightness width of 0.8 in latitude, while the higher latitude feature at $b=+1: 8$ has a half-brightness width of only 0.2 . A wide range of velocity dispersions is also observed, as can readily be seen in Figure 2, which shows a feature with a width between half intensity of nearly 30 $\mathrm{km} / \mathrm{sec}$, as well as a small, dense concentration with a width of only $6 \mathrm{~km} / \mathrm{sec}$.

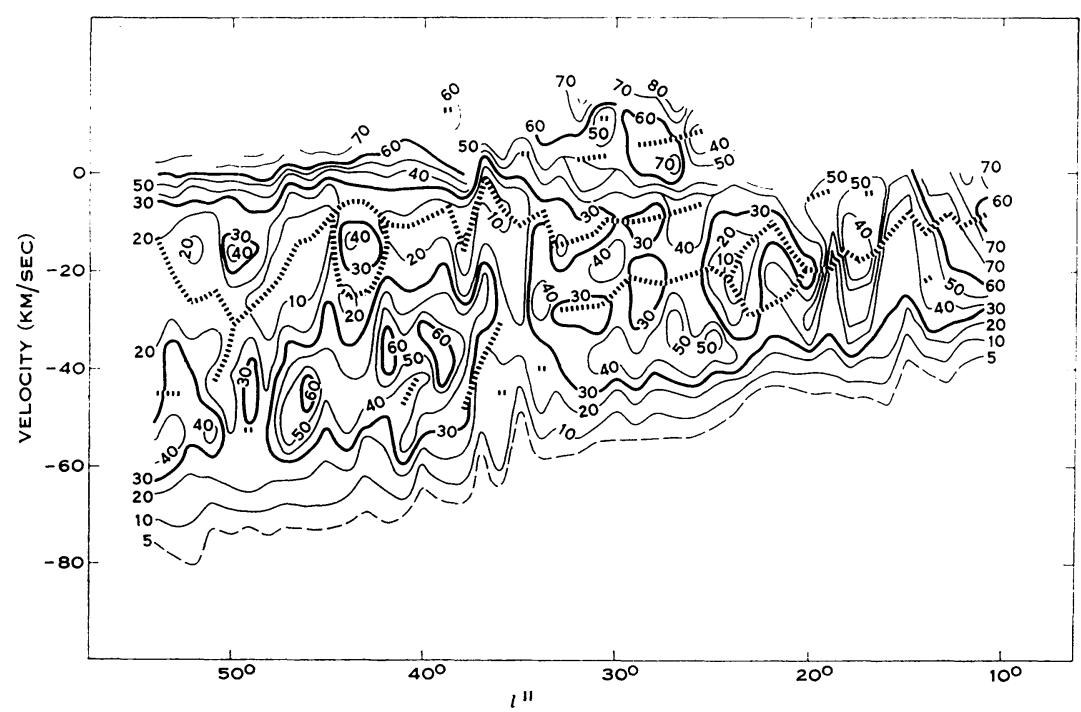

Fig. 3.-Observed antenna temperature along $b=+0.4$. Velocity is with respect to the local standard of rest.

(b) The angular extent of the hydrogen distribution above and below the plane was considerably greater than expected. The half-density thickness in $z$, when it can be defined, appears to be more than twice the thickness of the hydrogen in the inner parts of the Galaxy. The highest velocity (bluest) parts of the profiles vary only slightly over the entire range of latitudes studied. The $10^{\circ}$ isophote, for example, appears at nearly the same velocity, at a given longitude, from $b=-0.8$ to $+2^{\circ} .0$. There is little evidence of the drastic fluctuations observed in the peak intensities.

(c) The longitudinal map at $b=+0.4$ does show signs of being interpretable in terms of spiral structure. The hydrogen peaks, however, show considerable scatter, particularly for $l>30^{\circ}$. The valleys are somewhat more regular in their velocities, but the smoothest feature is the $10^{\circ}$ contour. Figure 4 shows the position of the $10^{\circ}$ contour on a plot of radial velocity versus $\sin l$. The line shown is a least-squares fit to the points. The radial velocity of this contour varies in remarkably linear fashion with $\sin l$, but intercepts the ordinate at about $20 \mathrm{~km} / \mathrm{sec}$, instead of going through the origin, as would be expected for purely circular motion. The lumpiness of the hydrogen maxima in Figure 3 is not mirrored in the $10^{\circ}$ contour, which suggests that 
we are dealing with separate systems. These observations indicate that the hydrogen in the outer parts of the Galaxy may be divided into two components: first, the dense hydrogen concentrations that are usually associated with spiral features, fluctuating widely in density and exhibiting small $(5-10 \mathrm{~km} / \mathrm{sec})$ internal velocity dispersion on a small scale, with larger deviations from strictly circular orbits on a large scale; and second, a thin substrate of gas with rather smooth distribution, exhibiting circular

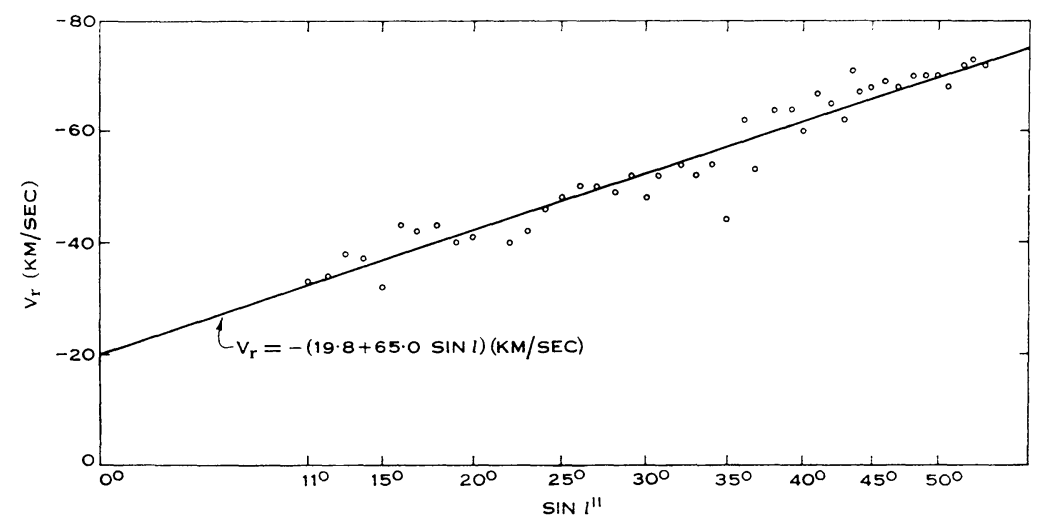

Fig. 4.-Velocity of the $10^{\circ} \mathrm{K}$ contour of antenna temperature with respect to the local standard of rest against $\sin l^{\mathrm{II}}(b=+0.4)$.

motion but with small-scale random motion of at least $20 \mathrm{~km} / \mathrm{sec}$. The substrate is observed over this longitude range on the negative-velocity side, with the random motions of the opposite sign being lost in the more intense radiation of the denser hydrogen concentrations, and hence only a rough estimate of the random internal motions can be given.

\section{Discussion}

Oort: In connection with the regular substratum of neutral hydrogen which Dr. Burke indicated, I wish to remark that Mr. Shane in Leiden found an indication of something that might be similar. He observed that in his section perpendicular to the Milky Way at $l^{\text {II }}$ between 22 and $42^{\circ}$ the outer contours were in general much smoother than the higher temperature contours. His outer contours, however, are much fainter than those considered by Dr. Burke, viz. between 2 and $4^{\circ} \mathrm{K}$.

\section{THE MAGNETIC FIELD OF THE GALAXY DETERMINED FROM THE ZEEMAN SPLITTING OF THE 21-CM HYDROGEN LINE}

\section{R. D. Davies}

\section{Nuffield Radio Astronomy Laboratories, Jodrell Bank}

The Zeeman effect can be used to measure directly the longitudinal component of the magnetic field in interstellar neutral hydrogen clouds. The frequency separation between the two circularly polarized components is $28 \mathrm{c} / \mathrm{s}$ for $10^{-5} \mathrm{G}$ and can be inferred from measurements of the intensity difference between left- and right-hand circular polarization as a function of frequency. Earlier experiments at Jodrell Bank 